\section{P2-104 ASSOCIATION OF NEIGHBOURHOOD SOCIOECONOMIC STATUS AND INDIVIDUAL SOCIOECONOMIC STATUS WITH CARDIOVASCULAR RISK FACTORS IN AN EASTERN GERMAN POPULATION - THE CARLA STUDY 2002-2006}

doi:10.1136/jech.2011.142976i.39

${ }^{1} \mathrm{~K}$ H Greiser, ${ }^{*} \mathrm{D}$ Tiller, ${ }^{2} \mathrm{O}$ Kuss, ${ }^{2} \mathrm{~A}$ Kluttig, ${ }^{3} \mathrm{G}$ Rudge, ${ }^{4} \mathrm{~B}$ Schumann, ${ }^{5} \mathrm{~K}$ Werdan, ${ }^{2} \mathrm{~J}$ Haerting. ${ }^{1}$ German Cancer Research Center (DKFZ), Division of Cancer Epidemiology, Heidelberg, Germany; ${ }^{2}$ Institute of Medical Epidemiology, Biostatistics, and Informatics, Martin-Luther-University Halle-Wittenberg, Halle (Saale), Germany; ${ }^{3}$ Public Health, Epidemiology and Biostatistics Unit, The College of Medical and Dental Sciences, The University of Birmingham, Birmingham, UK; ${ }^{4}$ Centre for Global Health Research, Umeå University, Umeå, Sweden; ${ }^{5}$ University Clinic for Internal Medicine III, Martin-Luther-University Halle-Wittenberg, Halle (Saale), Germany

Introduction/objectives Individual socioeconomic status (SES) is a determinant of cardiovascular risk factors (RF). Recent studies suggest an independent association of neighbourhood SES with cardiovascular RF, but the mechanisms have not fully been understood. Our aim was to assess the association of neighbourhood and individual SES with cardiovascular RF in an Eastern German population.

Methods We used cross-sectional data of 1779 participants aged 45-83 years of the population-based CARLA study. We calculated linear mixed models to assess the age-adjusted influence of neighbourhood-specific unemployment rates and individual SES on smoking, systolic blood pressure (SBP), and body mass index (BMI). Spatial dependencies within and between neighbourhoods were adjusted for by using ICAR models.

Results Neighbourhood-specific unemployment rates varied between 6.3 and $35.3 \%$. Per $1 \%$ increase in the neighbourhood's unemployment rate, the number of cigarettes smoked/day increased by 0.11 in men ( $95 \%$ CI 0.09 to 0.12 ) and 0.05 , (CI 0.04 to 0.07 ) in women. In women, SBP increased by $0.04 \mathrm{~mm} \mathrm{Hg}$ with unemployment rate (CI 0.03 to 0.06 ), while there was no statistically significant association of SBP with SES in men. BMI was only in women significantly associated with unemployment (increase in BMI per $1 \%$ increase in unemployment rate 0.04 (CI 0.02 to 0.05 )). Associations of RF with individual SES were stronger than with neighbourhood SES in multiple models.

Conclusions Our findings confirm the previously described association of neighbourhood SES with smoking independent of individual SES, while we found inconsistent associations with SBP and BMI. The neighbourhood environment may be more relevant for behavioural than for biomedical risk factors.

\section{P2-105 FACTORS ASSOCIATED WITH SHORT AND LONG SICKNESS ABSENTEEISM AMONG HEALTHCARE WORKERS}

doi:10.1136/jech.2011.142976i.40

${ }^{1} \mathrm{R}$ H Griep, ${ }^{*} \mathrm{R}$ C Ferreira, ${ }^{3} \mathrm{M}$ de Jesus Mendes da Fonseca, ${ }^{1} \mathrm{P}$ R Vasconcellos-Silva, ${ }^{1} \mathrm{~L}$ Rotenberg. ${ }^{1}$ IOC/Fiocruz, Rio de Janeiro, RJ, Brazil; ${ }^{2}$ EEAN/UFRJ, Rio de Janeiro, RJ, Brazil; ${ }^{3}$ ENSP/Fiocruz, Rio de Janeiro, RJ, Brazil

Introduction Sickness absenteeism is a complex and multifactorial phenomenon. The present paper studies the association between variables of different levels of determination and short/long sickness absenteeism reported by nursing staff

Methods Sectional study with 1509 workers (89.4\% of all workers) from three public hospitals in Rio de Janeiro, Brazil. Sickness absence was assessed by means of the self-reported question: "How many whole days have you been off work due to health problems (diseases or healthcare or for examination) during the past year (12 months)?" and we defined short sickness absenteeism (1-9 days) and long ( $\geq 10$ days). The logistic regression analysis used a conceptual model that consider distal (socio-economic conditions), intermediate I (occupational conditions), intermediate II (lifestyle characteristics) and proximal determinants (diseases and health conditions).

Results The prevalence of short and long absenteeism were respectively $20.3 \%$ and $16.6 \%$. In multivariate analysis, both types of absenteeism were associated with the role of practical nurse, musculoskeletal diseases, self-perceived poor/regular health and presence of minor psychological distress. In addition, short absenteeism was also associated with age and having more than one job, while long absenteeism was related to marital status and being a public servant.

Conclusion The results indicated variables of different levels of determination were linked to sickness absenteeism. Sickness absenteeism seems to indicate a multiple and complex determination that cannot be explained exclusively by health conditions.

\section{P2-106 SCREENING OF LUNG FUNCTION DISORDERS AMONG SMOKERS IN PRIMARY HEALTHCARE: A RUSSIAN STUDY}

doi:10.1136/jech.2011.142976i.41

${ }^{1}$ E Andreeva, ${ }^{1}$ I Kudryavtseva, ${ }^{2,3}$ A Grjibovski. ${ }^{1}$ International School of Public Health, Northern State Medical University, Arkhangelsk, Russia; ${ }^{2}$ Norwegian Institute of Public Health, Oslo, Norway; ${ }^{3}$ Institute of Community Medicine, University of Tromsø, Tromsø, Norway

Introduction Smoking is the main risk factor for chronic obstructive pulmonary disease (COPD). The prevalence of smoking in Russian Federation is one of the highest in the world. Many cases of COPD remain undiagnosed; therefore screening of COPD is an important task of the primary healthcare service. This study aims to estimate lung function disorders among smoking in primary healthcare in Northwest Russia.

Methods Altogether, 414 individuals, both smokers (89\%) and exsmokers $(11 \%)$ were invited and filled out questionnaires on smoking status and symptoms of COPD. Carboxyhaemoglobin (COHb\%) and COppm were measured with a smokelyzer. A standardised pulmonary function test with bronchodilatator for detection of bronchial obstruction were performed in smokers and ex-smokers, and patients with symptoms.

Results Mean age in the sample was 42.1 years (62.3\% were males). The average smoking history was 25 pack-years for men and 14.8 pack-years for women. High level of $\mathrm{COHb}(>3 \%)$ was detected in $35.9 \%$ of smokers. Linear regression analysis revealed positive association between $\mathrm{CO}$ and the number of cigarettes smoked per day ( $\mathrm{p}<0.001) .75 .4 \%$ had symptoms (cough, sputum and dyspnoea). COPD was detected in 28 individuals (6\%). [COPD I in 11; COPD II in 13 and COPD III in four individuals]

Conclusion Screening for COPD among smokers seems to be beneficial for early detection of COPD.

\section{P2-107 THYROID CANCER IN THE ARKHANGELSK REGION, RUSSIA} IN 2000-2009: EPIDEMIOLOGY AND SURVIVAL

doi:10.1136/jech.2011.142976i.42

${ }^{1} \mathrm{~N}$ Glukhareva, ${ }^{2} \mathrm{M}$ Valkov, ${ }^{3,4} \mathrm{~A}$ Grijbovski. ${ }^{1}$ International School of Public Health, Northern State Medical University, Arkhangelsk, Russia; ${ }^{2}$ Department of Radiology and Radiation Oncology with course of Clinical Oncology, Northern State Medical University, Arkhangelsk, Russia; ${ }^{3}$ Norwegian Institute of Public Health, Oslo, Norway: ${ }^{4}$ Institute of Community Medicine, University of Tromsø, Tromsø, Norway

Introduction The aim of the study is to assess the incidence of thyroid cancer in the Arkhangelsk region, Northwest Russia in 2000-2009 and to study factors influencing survival. 
Methods All incident cases of thyroid cancer (C73) in the Arkhangelsk region in 2000-2009 as well as information about deaths were extracted from the regional cancer registry. Population size was obtained from the Regional Bureau of Statistics. Incidence and mortality were calculated per 100 000. Survival was analysed using Kaplan-Meier curves with log rank tests.

Results Altogether, 529 new cases of thyroid cancer occurred in the region during the study period. Females comprised $82.8 \%$ of cases. Papillary carcinoma, follicular carcinoma and other forms was found in $56.3 \%, 27.7 \%$ and $21.9 \%$ of patients, respectively. Stages $0,1,2,3$, and 4 were diagnosed in $1.1 \%, 58.0 \%, 16.4 \%, 13.2 \%$, and $11.0 \%$ of patients respectively. The incidence increased from 4.3 in 2000 to 4.7 in 2009. Mortality decreased from 0.42 in 2000 to 0.24 in 2009. By 2010, 50 (9.5\%) died from thyroid cancer and 33 (6.2\%) were censored. Mean overall survival was 108 (95\% CI 105 to 111) months. Males, patients aged $45+$ years and those having stages 3-4 had significantly lower survival than the reference groups (all at $\mathrm{p}<0.001$ ). Average survival time for patients with papillary, follicular and other morphology types was 117, 107 and 83 months, respectively $(p<0.001)$

Conclusions Incidence of thyroid cancer in Arkhangelsk region of Russia slightly increased while the mortality decreased. Age, stage, morphology and gender were all associated with survival.

\section{P2-108 TRENDS IN MORTALITY FROM BREAST AND CERVICAL CANCER IN THE BRAZILIAN CAPITAL CITIES, 1996-2007}

doi:10.1136/jech.2011.142976i.43

${ }^{1} \mathrm{R}$ Guimarães, ${ }^{*}{ }^{2} \mathrm{C}$ Muzi, ${ }^{3} \mathrm{~A}$ Ayres, ${ }^{4} \mathrm{M}$ Ribeiro, ${ }^{4} \mathrm{C}$ Chagas, ${ }^{4} \mathrm{~J}$ Capilupi, ${ }^{4} \mathrm{P}$ Salles, ${ }^{4} \mathrm{Z}$ Melo, ${ }^{4} \mathrm{M}$ Matos. ${ }^{1}$ Federal University of Rio de Janeiro, Institute of Studies in Public Health, Rio de Janeiro, Brazil; ${ }^{2}$ National Cancer Institute, Rio de Janeiro, Brazil; ${ }^{3}$ Gaffrée Guinle University Hospital, Rio de Janeiro, Brazil;, ${ }^{4}$ Federal University of Rio de Janeiro, Rio de Janeiro, Brazil

Objective To analyse the trend in mortality from breast cancer and cervical cancer in Brazilian capitals from 1996 to 2007.

Materials and Methods We calculated mortality rates for breast cancer and cervical cancer in Brazilian capital cities from 1996 to 2007, standardised for the Brazilian population and for the world's population. Trend analysis was performed using the polynomial regression model.

Results Cancer of the cervix showed a considerable reduction in mortality rate in most capital cities, although in some cities the rate rose. Breast cancer mortality remained high in most of the capital cities. Trends were predominantly linear.

Conclusion It may be that mortality from cancer of the cervix may be failing due to increased adherence with Pap smear screening. The high breast cancer mortality rates may relate to its associations with habits, lifestyles and culture.

\section{P2-109 INCREASE INCOME AND MORTALITY OF COLORECTAL CANCER IN BRAZIL}

doi:10.1136/jech.2011.142976i.44

${ }^{1} \mathrm{R}$ Guimarães, ${ }^{*} \mathrm{C}$ Muzi, ${ }^{2} \mathrm{R}$ Ramos, ${ }^{3} \mathrm{~S}$ Annes, ${ }^{4} \mathrm{C}$ Queiróz. ${ }^{1}$ Federal University of Rio de Janeiro, Institute of Studies in Public Health, Rio de Janeiro, Brazil; ${ }^{2}$ National Cancer Institute, Rio de Janeiro, Brazil; ${ }^{3}$ Rio de Janeiro State Health Department, Rio de Janeiro, Brazil; ${ }^{4}$ Federal University of Rio de Janeiro, Rio de Janeiro, Brazil

Objective The objective of this study is to determine the correlation between average per capita income and the rate of colorectal cancer mortality in Brazil between 2000 and 2007.

Materials and Methods We obtained data on median household income and mortality rate for colon, rectum and anus between 2001 and 2007 by DATASUS. A trend analysis was performed using linear regression, and correlation between variables by Pearson's correlation coefficient.

Results There was a tendency towards an increase average family income and standardised mortality rate of colon, rectum and anus in Brazil. There was also strong positive correlation $(r=0.81, p<0.001)$ between income and mortality for this cancer located throughout the study period.

Conclusion The increase in income may partially explain the increased occurrence of cancer of the colon, rectum and anus, and this is possibly due to differential access to food recognised as a risk factor, such as red meat and fat food. É therefore important Assess the priority of public health programs addressing nutrition in countries with intermediate economy, as is the case in Brazil.

\section{P2-110 NUMBER OF TEETH IS ASSOCIATED WITH THE LEVEL OF SALIVARY NITRIC OXIDE}

doi:10.1136/jech.2011.142976i.45

D-H Han, ${ }^{*}$ H-D Kim, H-M Kim, H-S Shin, M-S Kim. Seoul National University, Seoul, Republic of Korea

Introduction Nitric oxide (NO) is involved in second messenger formation, osteoblast and osteoclast function, and blood flow. This raises the question of whether or not altered salivary NO levels interfere with tooth loss by influencing the bone remodelling cycle. This study investigates if different levels of salivary NO are associated with existing number of teeth among Korean elderly.

Methods A total of 178 subjects (68 males and 110 females; age range 48-84 years) received a dental and general examination in 2009 and 2010. Number of remaining teeth and salivary flow rate were evaluated by a dentist. Fasting plasma glucose level and blood pressure were also measured. Age, gender, education level, and smoking were evaluated through interview. Salivary NO was determined by the Griess reagent. ANCOVA and Multiple linear regression analyses were applied.

Results After controlling for age, gender, education level, smoking, physical activity, salivary flow rate, fasting plasma glucose level, systolic blood pressure and diastolic blood pressure, higher NO level group showed increased number of teeth compared to moderate and lower NO level group, which is not significant. However, salivary NO levels had a dose-response relationship with the existing number of teeth in Korean elderly $(\beta=-0.007, p=0.046)$.

Conclusion This study presents for the first time a dose-dependent relationship between salivary NO levels and number of teeth, indicating a link between oral health and salivary $\mathrm{NO}$ levels, and that the levels of salivary $\mathrm{NO}$ could be a proper indicator for oral health in this respect.

\section{P2-111 IMPROVING THE HEALTH OF JAPANESE WOMEN: ACCEPTANCE OF HPV VACCINATION IN MOTHERS OF ADOLESCENT GIRLS}

doi:10.1136/jech.2011.142976i.46

${ }^{1,2} \mathrm{~S}$ Hanley, ${ }^{*}{ }^{1} \mathrm{E}$ Yoshioka, ${ }^{2} \mathrm{Y}$ Ito, ${ }^{3} \mathrm{R}$ Konno, ${ }^{4} \mathrm{~N}$ Sakuragi, ${ }^{5} \mathrm{R}$ Kishi. ${ }^{1}$ Hokkaido University, Graduate School of Medicine, Department of Public Health, Sapporo, Japan; ${ }^{2}$ Japanese Red Cross Hokkaido College of Nursing, Faculty of Nursing, Kitami, Japan; ${ }^{3}$ Jichi Medical University, Saitama Medical Center, Department of Obstetrics and Gynecology, Saitama, Japan; ${ }^{4}$ Hokkaido University, Graduate School of Medicine, Department of Reproductive Endocrinology and Oncology, Sapporo, Japan; ${ }^{5}$ Hokkaido University, Center for Environmental and Health Sciences, Sapporo, Japan

Introduction Cervical Cancer (CC) caused by oncogenic Human Papillomavirus (HPV) is the most common cancer in young Japanese 